\title{
DIRICHLET BOUNDARY VALUE PROBLEM FOR A THIRD ORDER PARABOLIC-HYPERBOLIC EQUATION WITH DEGENERATING TYPE AND ORDER IN THE HYPERBOLICITY DOMAIN
}

\author{
Zh.A. BALKIZOV
}

\begin{abstract}
In the work we study an analogue of Tricomi equation for a third order parabolic-hyperbolic equation with smaller derivatives having multiple characteristics. Under certain conditions for the given functions and parameters involved in the considered equation, we prove unique solvability theorem for the studied problem. The uniqueness of the solution is proved by means of the generalized Tricomi method, while the existence is proved via the method of integral equations.
\end{abstract}

Keywords: Degenerate hyperbolic equation, equation with multiple characteristics, third order parabolic-hyperbolic equation, Dirichlet boundary value problem, analogue of Tricomi equation, Tricomi method, second kind integral Volterra equation, second kind integral Fredholm equation.

\section{Mathematics Subject Classification: $35 \mathrm{M} 12$}

\section{INTRODUCTION}

In the Euclidean plane of independent variables $x$ and $y$ we consider the equation

$$
0= \begin{cases}(-y)^{m} u_{x x}-u_{y y}+a(-y)^{(m-2) / 2} u_{x}, & y<0, \\ u_{x x x}-u_{y}+\sum_{i=0}^{2} a_{i}(x, y) \frac{\partial^{i} u}{\partial x^{i}}, & y>0,\end{cases}
$$

where $a_{i}(x, y), i=\overline{0,2}$, are given functions; $a, m$ are given numbers and $m>0,|a| \leqslant m / 2$; $u=u(x, y)$ is the unknown function.

By $\Omega$ we denote the domain as $y<0$ bounded by the characteristics

$$
A C: x-\frac{2}{m+2}(-y)^{(m+2) / 2}=0 \quad \text { and } \quad C B: x+\frac{2}{m+2}(-y)^{(m+2) / 2}=r
$$

of equation (1.1) leaving the points $A=(0,0), B=(r, 0)$, intersecting at the point $C=$ $\left(r / 2, y_{c}\right), y_{c}<0$, and as $y>0$, this domain is the rectangle with the vertices at the points $A$, $B, A_{0}=(0, h)$ and $B_{0}=(r, h), h>0$. We also let $\Omega_{1}=\Omega \cap\{y<0\}, \quad \Omega_{2}=\Omega \cap\{y>0\}$, $\Omega=\Omega_{1} \cup \Omega_{2} \cup J$, where $J=\{(x, 0): 0<x<r\}$ is the interval $A B$ of the line $y=0$.

As $y<0$, equation (1.1) coincides with the degenerate hyperbolic equation

$$
(-y)^{m} u_{x x}-u_{y y}+a(-y)^{(m-2) / 2} u_{x}=0
$$

Zh.A. BAlkizov, Dirichlet BOUNDARY VAlUe PROBlem FOR A THIRD ORDER PARABOLIC-HYPERBOLIC EQUATION WITH DEGENERATING TYPE AND ORDER IN THE HYPERBOLICITY DOMAIN.

(C) BALKizOV ZH.A. 2017.

Submitted July 7, 2016. 
and as $y>0$, this is the third order equation of the form

$$
u_{x x x}-u_{y}+\sum_{i=0}^{2} a_{i}(x, y) \frac{\partial^{i} u}{\partial x^{i}}=0 .
$$

Equation 1.2 is an equation of hyperbolic type with a parabolic degeneration along the line $y=0$. As $m=2$, equation (1.2) becomes Bitsadze-Lykov equation [1], [2], [3], and as $a=0$, equation (1.2) leads us to the Gellerstedt equation and as it was shown in [4], this equation has application in the problem on determining the shape of the cut in a weir. A particular case of equation (1.2) is the Tricomi equation being a theoretical base of transsonic gas dynamic [5], [6]. Works [7], [8] were devoted to studying the Darboux first and second problem for equation (1.2). In work [9] there was studied a criterion of the continuity of a solution to the Goursat problem for a degenerate hyperbolic equation of form (1.2). A rather complete bibliography on studying various boundary value problems for the degenerate hyperbolic equation was provided in monographs [10]-[13].

Equation (1.3) called in [14] the third order equation with multiple characteristics is a parabolic equation [3]. The study of boundary value problems for equations of form (1.3) was initiated by the results of work [15], where a boundary value problem nowadays called Cattabriga problem, was studied by means of the methods of the potential theory and by the integral Laplace transform. By means of the fundamental solutions of equation (1.3) obtained in [15], in [14] there was constructed the Green function for the Cattabriga problem for equation (1.3) and there were obtained the estimates for the fundamental solutions and their derivatives of various orders. Also, by means of the Green function, in [14] there was constructed a solution of Cattabriga problem for equation (1.3) in a closed form. Works [16-18] were devoted to studying various local and nonlocal boundary value problems for equation (1.3).

Equation (1) belongs to the class of third order equations of parabolic-hyperbolic type with a degeneration along the line $y=0$ of the type changing. The need of considering the problem on matching parabolic and hyperbolic equations was observed first in work [19]. The problem on matching parabolic and hyperbolic equations arises in studying electric oscillations in wires. Such problems also arise in studying the motion of a liquid in channel enveloped by a porous media, in the theory of electromagnetic waves propagation and in a series of other field in the physics.

The importance of studying boundary value problems for the high order mixed type equations was pointed out in work [20] and it was mentioned in work [21] that an order degeneration along the line of type changing brings new aspects in the theory of mixed type equations. The topicality of studying well-posed boundary value problems for high order mixed type equations is supported also by numerous publications in this direction by domestic and foreign authors. For instance, for a model third order parabolic-hyperbolic equation with the Gellerstedt equation in the hyperbolicity domain, in work [22] there was studied a nonlocal interior boundary value problem with a shift involving Saigo operator in boundary conditions, while in work [23] there was studied a similar problem for equation of form $(1.1)$ with $a_{2}(x, y) \equiv 0$. Boundary value problem for third order parabolic-hyperbolic equations with various degenerating operators in hyperbolicity domain were studied in [24]-[28].

In view of the said above, there arises a need on finding well-posed boundary value problems posed simultaneously for degenerating hyperbolic equations and high order equations with multiple characteristics. In the present work we study an analogue of Tricomi problem for the third order parabolic-hyperbolic equations with a degeneration of type and order in the hyperbolicity domain. Among early works closely related with the present work, we distinguish works $[29,30]$, where there was studied the well-posedness of the matching problem for model and general parabolic and hyperbolic equations in time variable and there were studied structural and qualitative properties of their solutions. 


\section{Formulation of the PROBLEM AND MAIN RESUlts}

A regular in the domain $\Omega$ solution to equation (1.1) is a function $u=u(x, y)$ in the class $C(\bar{\Omega}) \cap C^{1}(\Omega) \cap C^{2}\left(\Omega_{1}\right) \cap C_{x}^{3}\left(\Omega_{2}\right), u_{x}(x, 0), u_{y}(x, 0) \in L_{1}(0, r)$, such that substituting this function into equation $(1.1)$, we get the identity.

In the work we study the following

Problem 1. Find a regular in the domain $\Omega$ solution to equation (1.1) satisfying the conditions

$$
\begin{aligned}
& u(0, y)=\varphi_{1}(y), \quad u_{x}(0, y)=\varphi_{2}(y), \quad u(r, y)=\varphi_{3}(y), \quad 0 \leqslant y<h \\
& \left.u\right|_{C B}=\psi(x), \quad \frac{r}{2} \leqslant x \leqslant r
\end{aligned}
$$

where $\varphi_{1}(y), \varphi_{2}(y), \varphi_{3}(y) \in C[0, h], \psi(x) \in C^{1}[r / 2, r]$ are given functions.

The main aim of the present is to prove the theorem on the unique solvability of Problem 1.

\section{UNIQUENESS THEOREM}

We denote

$$
\alpha=\frac{m-2 a}{2(m+2)}, \quad \beta=\frac{m+2 a}{2(m+2)}, \quad \gamma_{1}=\frac{2 \Gamma(1-\beta) \Gamma(\alpha+\beta)}{\Gamma(\alpha) \Gamma(1-\alpha-\beta)[2(1-\alpha-\beta)]^{\alpha+\beta}} .
$$

The following theorem holds true.

Theorem 3.1. Assume that the coefficients $a_{i}(x, y), i=\overline{0,2}$, of equation (1.1) satisfy the conditions:

$$
\begin{aligned}
& a_{i}(x, y) \in C^{i}\left(\bar{\Omega}_{2}\right), \quad i=\overline{0,2} \\
& a_{2}(x, 0) \geqslant 0, \quad 0 \leqslant x \leqslant r \\
& x^{1-\alpha-\beta}\left[a_{2}^{\prime \prime}(x, 0)-a_{1}^{\prime}(x, 0)+2 a_{0}(x, 0)\right] \leqslant \frac{\gamma_{1}}{\Gamma(\alpha+\beta)}, \quad 0<x<r, \\
& a_{2}^{2}(x, 0)+\left[a_{2}^{\prime \prime}(x, 0)-a_{1}^{\prime}(x, 0)+2 a_{0}(x, 0)-\frac{\gamma_{1}}{\Gamma(\alpha+\beta)} x^{\alpha+\beta-1}\right]^{2}>0, \quad 0<x<r .
\end{aligned}
$$

Then a solution to Problem 1 in the domain $\Omega$ is unique.

Proof. To prove the theorem, we introduce the notations:

$$
\begin{array}{ll}
u(x, 0)=\tau(x), & 0 \leqslant x \leqslant r, \\
u_{y}(x, 0)=\nu(x), & 0<x<r .
\end{array}
$$

Regarding the functions $\tau(x)$ and $\nu(x)$ as given, let us write the solution to Cauchy problem (3.5) - 3.6) for equation 1.2 .

We suppose first that $|a|<\frac{m}{2}$. In this case a solution to problem $(3.5)-(3.6)$ for equation (1.2) is written out by the formula [10]:

$$
\begin{aligned}
u(x, y)= & \frac{1}{B(\alpha, \beta)} \int_{0}^{1} \tau\left[x+\frac{2}{m+2}(-y)^{(m+2) / 2}(2 t-1)\right] t^{\beta-1}(1-t)^{\alpha-1} d t \\
& +\frac{y}{B(1-\alpha, 1-\beta)} \int_{0}^{1} \nu\left[x+\frac{2}{m+2}(-y)^{(m+2) / 2}(2 t-1)\right] t^{-\alpha}(1-t)^{-\beta} d t,
\end{aligned}
$$

where $B(p, q)$ is the first kind Euler integral (beta function). 
Substituting (2.2) into satisfy condition (3.7), we find:

$$
\begin{aligned}
\left.u(x, y)\right|_{C B}= & \frac{1}{B(\alpha, \beta)} \int_{0}^{1} \tau[x+(r-x)(2 t-1)] t^{\beta-1}(1-t)^{\alpha-1} d t \\
& -\frac{(1-\alpha-\beta)^{\alpha+\beta-1}}{B(1-\alpha, 1-\beta)}(r-x)^{1-\alpha-\beta} \int_{0}^{1} \nu[x+(r-x)(2 t-1)] t^{-\alpha}(1-t)^{-\beta} d t \\
= & \psi(x) .
\end{aligned}
$$

Changing first the integration variable $s=2 x-r+2 r t-2 x t$, and then replacing $2 x-r$ by $x$ in the obtained identity, we rewrite the latter relation as

$$
\begin{aligned}
\frac{(r-x)^{1-\alpha-\beta}}{B(\alpha, \beta)} & \int_{x}^{r} \tau(t)(r-t)^{\alpha-1}(t-x)^{\beta-1} d t \\
& -\frac{[2(1-\alpha-\beta)]^{\alpha+\beta-1}}{B(1-\alpha, 1-\beta)} \int_{x}^{r} \nu(t)(r-t)^{-\beta}(t-x)^{-\alpha} d t=\psi\left(\frac{r+x}{2}\right) .
\end{aligned}
$$

Now we employ the following definition of the fractional integro-differentiation [3]: the operator of fractional (in the Riemann-Liouville sense) integro-differentiation of order $|\alpha|$ with the origin at the point $c \in[a, b]$ is the operator $D_{c x}^{\alpha}$ acting on an absolutely integrable function $\varphi(t) \in$ $L_{1}(a, b)$ by the formula:

$$
\begin{aligned}
& D_{c x}^{\alpha} \varphi(t)=\frac{\operatorname{sgn}(x-c)}{\Gamma(-\alpha)} \int_{c}^{x}|x-t|^{-(\alpha+1)} \varphi(t) d t, \quad \alpha<0, \\
& D_{c x}^{\alpha} \varphi(t)=\operatorname{sgn}^{[\alpha]+1}(x-c) \frac{d^{[\alpha]+1}}{d x^{[\alpha]+1}} D_{c x}^{\alpha-[\alpha]-1} \varphi(t), \quad \alpha>0,
\end{aligned}
$$

where the $\operatorname{symbol} \operatorname{sgn}(z)$ stands for the sign of a number $z, \Gamma(x)$ is the second kind Euler integral (Gamma function). A detailed study of the properties of the operator $D_{c x}^{\alpha} \varphi(t)$ were provided in monographs [3], [4], [31].

In view of the above definition of the operator $D_{c x}^{\alpha}$, relation $(3.8)$ is rewritten as

$$
\begin{aligned}
& \frac{\Gamma(\beta)}{B(\alpha, \beta)}(r-x)^{1-\alpha-\beta} D_{r x}^{-\beta}\left[\tau(t)(r-t)^{\alpha-1}\right] \\
& -\frac{\Gamma(1-\alpha)[2(1-\alpha-\beta)]^{\alpha+\beta-1}}{B(1-\alpha, 1-\beta)} D_{r x}^{\alpha-1}\left[(r-t)^{-\beta} \nu(t)\right]=\psi\left(\frac{r+x}{2}\right) .
\end{aligned}
$$

Solving equation $(3.9)$ w.r.t. the function $\nu(x)$, we find

$$
\nu(x)=\gamma_{1} D_{r x}^{1-\alpha-\beta} \tau(t)-\gamma_{2}(r-x)^{\beta} D_{r x}^{1-\alpha}\left[\psi\left(\frac{t+r}{2}\right)\right],
$$

where

$$
\gamma_{2}=\frac{2 \Gamma(1-\beta)}{\Gamma(1-\alpha-\beta)[2(1-\alpha-\beta)]^{\alpha+\beta}} .
$$

Since $\tau(x), \psi\left(\frac{r+x}{2}\right) \in C[0, r]$, and $\tau^{\prime}(x), \psi^{\prime}\left(\frac{r+x}{2}\right) \in L_{1}(0, r)$, then employing the following property of the fractional integro-differentiation operator of order $0<\alpha \leqslant 1$ [31]

$$
D_{r x}^{\alpha} \varphi(t)=\frac{\varphi(r)}{\Gamma(1-\alpha)}(r-x)^{-\alpha}-D_{r x}^{\alpha-1} \varphi^{\prime}(t)
$$


we can rewrite expression $(3.10)$ as

$$
\nu(x)=-\gamma_{1} D_{r x}^{-(\alpha+\beta)} \tau^{\prime}(t)+\frac{\gamma_{2}}{2}(r-x)^{\beta} D_{r x}^{-\alpha} \psi^{\prime}\left(\frac{r+t}{2}\right) .
$$

Relation 3.12 is a fundamental one for the sought functions $\tau(x)$ and $\nu(x)$ moved from the domain $\Omega_{1}$ on the line $y=0$ in the case $|a|<\frac{m}{2}$.

If $a=-\frac{m}{2}$, then $\alpha=\frac{m}{m+2}, \beta=0$ and a solution to problem (3.5)-3.6 for equation (1.2) is of the form $[10]$ :

$$
\begin{aligned}
u(x, y)= & \tau\left[x+\frac{2}{m+2}(-y)^{(m+2) / 2}\right] \\
& +\frac{2 y}{m+2} \int_{0}^{1} \nu\left[x+\frac{2}{m+2}(-y)^{(m+2) / 2}(2 t-1)\right](1-t)^{-\alpha} d t .
\end{aligned}
$$

By representation (3.13) and in view of condition (2.2), we arrive at a fundamental relation for the functions $\tau(x)$ and $\nu(x)$ :

$$
\nu(x)=-\frac{\gamma_{1}}{2}\left[2 D_{r x}^{-\alpha} \tau^{\prime}(t)-D_{r x}^{-\alpha} \psi^{\prime}\left(\frac{r+t}{2}\right)\right] .
$$

If $a=\frac{m}{2}$, then $\alpha=0, \beta=\frac{m}{m+2}$. In this case a solution to problem (3.5), (3.6) for equation 1.2) is of the form [10]:

$$
\begin{aligned}
u(x, y)= & \tau\left[x-\frac{2}{m+2}(-y)^{(m+2) / 2}\right] \\
& +\frac{2 y}{m+2} \int_{0}^{1} \nu\left[x-\frac{2}{m+2}(-y)^{(m+2) / 2}(2 t-1)\right](1-t)^{-\beta} d t .
\end{aligned}
$$

Satisfying boundary condition 2.2 on the characteristics $C B$ for $(3.15)$, we arrive at the identity

$$
\nu(x)=(2-2 \beta)^{-\beta}(r-x)^{\beta} \psi^{\prime}\left(\frac{r+x}{2}\right) .
$$

We proceed to proving the uniqueness of solution to Problem 1. For the homogeneous problem corresponding to Problem 1 we consider the integral

$$
J^{*}=\int_{0}^{r} \tau(x) \nu(x) d x .
$$

As $\psi(x) \equiv 0(\tau(r)=\psi(r)=0)$, by relations 3.12, 3.14), 3.16) for different values of $a$ we obtain the corresponding identities:

$$
\begin{aligned}
& \nu(x)=-\gamma_{1} D_{r x}^{-(\alpha+\beta)} \tau^{\prime}(t)=\gamma_{1} D_{r x}^{1-\alpha-\beta} \tau(t), \quad|a|<\frac{m}{2} \\
& \nu(x)=-\gamma_{1} D_{r x}^{-\alpha} \tau^{\prime}(t)=\gamma_{1} D_{r x}^{1-\alpha} \tau(t), \quad a=-\frac{m}{2} ; \\
& \nu(x) \equiv 0, \quad a=\frac{m}{2} .
\end{aligned}
$$

We employ the following property of the operator $D_{r x}^{\alpha} \varphi(t)$ of a fractional integrodifferentiation (in the Riemann-Liouville sense). 
Lemma 3.1. For an absolutely continuous on the segment $[0, r]$ function $\varphi=\varphi(x)$ satisfying the condition $\varphi(r)=0$ the inequality

$$
\varphi(x) D_{r x}^{\alpha} \varphi(t) \geqslant \frac{1}{2} D_{r x}^{\alpha} \varphi^{2}(t), \quad 0<\alpha \leqslant 1,
$$

holds true.

Proof. Indeed, if $\varphi(r)=0$, by Formula 3.11 we find

$$
D_{r x}^{\alpha} \varphi(t)=-\frac{1}{\Gamma(1-\alpha)} \int_{x}^{r} \frac{\varphi^{\prime}(t)}{(t-x)^{\alpha}} d t
$$

In the same way,

$$
D_{r x}^{\alpha} \varphi^{2}(t)=-\frac{1}{\Gamma(1-\alpha)} \int_{x}^{r} \frac{2 \varphi(t) \varphi^{\prime}(t)}{(t-x)^{\alpha}} d t .
$$

Employing the above identities, we find

$$
\begin{aligned}
\varphi(x) D_{r x}^{\alpha} \varphi(t)-\frac{1}{2} D_{r x}^{\alpha} \varphi^{2}(t) & =\frac{1}{\Gamma(1-\alpha)} \int_{x}^{r} \frac{\varphi^{\prime}(t)[\varphi(t)-\varphi(x)]}{(t-x)^{\alpha}} d t \\
& =\frac{1}{\Gamma(1-\alpha)} \int_{x}^{r} \frac{\varphi^{\prime}(t)}{(t-x)^{\alpha}}\left(\int_{x}^{t} \varphi^{\prime}(s) d s\right) d t \\
& =\frac{1}{\Gamma(1-\alpha)} \int_{x}^{r}\left(\int_{s}^{r} \frac{\varphi^{\prime}(t) \varphi^{\prime}(s)}{(t-x)^{\alpha}} d t\right) d s \\
& =\frac{1}{\Gamma(1-\alpha)} \int_{x}^{r}(s-x)^{\alpha} \frac{\varphi^{\prime}(s)}{(s-x)^{\alpha}}\left(\int_{s}^{r} \frac{\varphi^{\prime}(t)}{(t-x)^{\alpha}} d t\right)^{r} d s \\
& =-\frac{1}{2 \Gamma(1-\alpha)} \int_{x}^{r}(s-x)^{\alpha} \frac{\partial}{\partial s}\left[\left(\int_{s}^{r} \frac{\varphi^{\prime}(t)}{(t-x)^{\alpha}} d t\right)^{2}\right] d s \\
& =\frac{\alpha}{2 \Gamma(1-\alpha)} \int_{x}^{r}(s-x)^{\alpha-1}\left(\int_{s}^{r} \frac{\varphi^{\prime}(t)}{(t-x)^{\alpha}} d t\right)^{2} d s \geqslant 0,
\end{aligned}
$$

that implies inequality 3.20 . The proof is complete.

We note that the proven Lemma 3.1 is an analogue of Lemma 1 in work [32].

As $|a|<\frac{m}{2}$, by (3.17) and (3.20) we arrive at the inequality

$$
J^{*}=\gamma_{1} \int_{0}^{r} \tau(x) D_{r x}^{1-\alpha-\beta} \tau(t) d x \geqslant \frac{\gamma_{1}}{2 \Gamma(\alpha+\beta)} \int_{0}^{r} t^{\alpha+\beta-1} \tau^{2}(t) d t .
$$

We arrive at a similar inequality as $a=-\frac{m}{2}\left(\alpha=\frac{m}{m+2}, \beta=0\right)$, while as $a=\frac{m}{2}$ by identity (3.19) we obtain that $J^{*} \equiv 0$.

Passing then to the limit as $y \rightarrow+0$ in equation (1.1), in view of boundary conditions (2.1) we obtain a fundamental relation for the functions $\tau(x)$ and $\nu(x)$ moved from the parabolic part $\Omega_{2}$ of the domain $\Omega$ on the line $y=0$ :

$$
\nu(x)=\tau^{\prime \prime \prime}(x)+a_{2}(x, 0) \tau^{\prime \prime}(x)+a_{1}(x, 0) \tau^{\prime}(x)+a_{0}(x, 0) \tau(x), \quad 0<x<r,
$$




$$
\tau(0)=\varphi_{1}(0), \quad \tau^{\prime}(0)=\varphi_{2}(0), \quad \tau(r)=\varphi_{3}(0) .
$$

Lemma 3.2. Assume that conditions (3.1). Then by (3.22)-(3.23) we have the identity

$$
J^{*}=-\frac{\tau^{\prime 2}(r)}{2}-\int_{0}^{r} a_{2}(x, 0) \tau^{\prime 2}(x) d x+\frac{1}{2} \int_{0}^{r}\left[a_{2}^{\prime \prime}(x, 0)-a_{1}^{\prime}(x, 0)+2 a_{0}(x, 0)\right] \tau^{2}(x) d x .
$$

For homogeneous boundary conditions corresponding to conditions 3.23$)\left(\varphi_{j}(0)=0, j=\right.$ $\overline{1,3}$ ) identity (3.24) can be obtained easily by multiplying both sides of relation (3.22) by the function $\tau(x)$ and integrating then the obtained identity w.r.t. $x$ from 0 to $r$.

In view of (3.24), inequality (3.21) can be rewritten as

$$
\begin{aligned}
\tau^{\prime 2}(r) & +2 \int_{0}^{r} a_{2}(x, 0) \tau^{\prime 2}(x) d x \\
& -\int_{0}^{r}\left[a_{2}^{\prime \prime}(x, 0)-a_{1}^{\prime}(x, 0)+2 a_{0}(x, 0)-\frac{\gamma_{1}}{\Gamma(\alpha+\beta)} x^{\alpha+\beta-1}\right] \tau^{2}(x) d x \leqslant 0 .
\end{aligned}
$$

It is easy to see that under assumptions (3.2)-(3.4) of Theorem 3.1 for the coefficients $a_{i}(x, y), i=\overline{0,2}$ of equation (1.1), inequality (3.25) can hold if and only if $\tau(x) \equiv 0$. Then by relations (3.17), (3.18), (3.19) we find that $\nu(x) \equiv 0$ for all $|a| \leqslant \frac{m}{2}$. At that, formulae (3.7), 3.13, 3.15) imply immediately that $u(x, y) \equiv 0$ in $\overline{\Omega_{1}}$.

Let us show that under the assumptions of Theorem 3.1, the problem on finding a regular in the domain $\Omega_{2}$ solution of equation (1.3) satisfying homogeneous boundary conditions corresponding conditions (2.1) and the homogeneous initial condition $u(x, 0)=0$ can have only the trivial solution.

Indeed, assume that the homogeneous problem

$$
\begin{aligned}
& u_{x x x}-u_{y}+\sum_{i=0}^{2} a_{i}(x, y) \frac{\partial^{i} u}{\partial x^{i}}=0, \quad(x, y) \in \Omega_{2}, \\
& u(0, y)=0, u_{x}(0, y)=0, u(r, y)=0, \quad 0<y<h, \\
& u(x, 0)=0, \quad 0 \leqslant x \leqslant r,
\end{aligned}
$$

has a non-trivial solution $u=u(x, y) \not \equiv 0$. Following works [16], [33], in equation (3.26) we let

$$
u(x, y)=v(x, y) \exp \left(\mu_{1} x+\mu_{2} y\right) .
$$

Then for the function $v=v(x, y)$ we obtain the equation

$$
\begin{aligned}
L_{\mu_{1}, \mu_{2}} v= & v_{x x x}-v_{y}+\left[3 \mu_{1}+a_{2}(x, y)\right] v_{x x}+\left[3 \mu_{1}^{2}+2 \mu_{1} a_{2}(x, y)+a_{1}(x, y)\right] v_{x} \\
& +\left[\mu_{1}^{3}+\mu_{1}^{2} a_{2}(x, y)+\mu_{1} a_{1}(x, y)+a_{0}(x, y)-\mu_{2}\right] v=0
\end{aligned}
$$

subject to the initial and boundary conditions

$$
\begin{aligned}
& v(x, 0)=0, \quad 0 \leqslant x \leqslant r, \\
& v(0, y)=0, v_{x}(0, y)=0, v(r, y)=0, \quad 0<y<h .
\end{aligned}
$$

Since by the assumption the function $u(x, y)$ is a non-trivial solution of problem $(3.26)-(3.28)$, as it follows from (3.29), problem $(3.30)-3.32$ ) also has a non-trivial solution $v=v(x, y) \neq 0$.

We introduce an auxiliary domain $\Omega_{2 \varepsilon}$ by the inequalities

$$
\Omega_{2 \varepsilon}=\{(x, y): \varepsilon<x<r-\varepsilon, \varepsilon<y<h-\varepsilon, \varepsilon>0\} .
$$


In the domain $\Omega_{2 \varepsilon}$, the identity

$$
\begin{aligned}
2\left(v, L_{\mu_{1}, \mu_{2}} v\right)_{0}= & \int_{\Omega_{2 \varepsilon}} 2 v L_{\mu_{1}, \mu_{2}} v d \Omega_{2 \varepsilon} \\
= & \int_{\Omega_{2 \varepsilon}}\left\{\frac { \partial } { \partial x } \left[2 v v_{x x}-v_{x}^{2}+2\left(3 \mu_{1}+a_{2}(x, y)\right) v v_{x}\right.\right. \\
& \left.\left.+\left(3 \mu_{1}^{2}+2 \mu_{1} a_{2}(x, y)-a_{2 x}(x, y)+a_{1}(x, y)\right) v^{2}\right]-\frac{\partial}{\partial y}\left[v^{2}\right]\right\} d \Omega_{2 \varepsilon} \\
& +\int_{\Omega_{2 \varepsilon}}\left[2 \mu_{1}^{3}+2 \mu_{1}^{2} a_{2}(x, y)+2 \mu_{1} a_{1}(x, y)+a_{2 x x}(x, y)\right. \\
& \left.-a_{1 x}(x, y)+2 a_{0}(x, y)-2 \mu_{2}\right] v^{2} d \Omega_{2 \varepsilon} \\
& -2 \int_{\Omega_{2 \varepsilon}}\left[3 \mu_{1}+a_{2}(x, y)\right] v_{x}^{2} d \Omega_{2 \varepsilon}=0
\end{aligned}
$$

Applying Green formula to identity (3.33), we obtain

$$
\begin{aligned}
2\left(v, L_{\mu_{1}, \mu_{2}} v\right)_{0}= & \int_{\Gamma_{2 \varepsilon}} v^{2} d x+\left[2 v v_{x x}-v_{x}^{2}+2\left(3 \mu_{1}+a_{2}(x, y)\right) v v_{x}\right. \\
& \left.+\left(3 \mu_{1}^{2}+2 \mu_{1} a_{2}(x, y)-a_{2 x}(x, y)+a_{1}(x, y)\right) v^{2}\right] d y \\
+ & \int_{\Omega_{2 \varepsilon}}\left[2 \mu_{1}^{3}+2 \mu_{1}^{2} a_{2}(x, y)+2 \mu_{1} a_{1}(x, y)+a_{2 x x}(x, y)\right. \\
& \left.-a_{1 x}(x, y)+2 a_{0}(x, y)-2 \mu_{2}\right] v^{2} d \Omega_{2 \varepsilon} \\
- & 2 \int_{\Omega_{2 \varepsilon}}\left[3 \mu_{1}+a_{2}(x, y)\right] v_{x}^{2} d \Omega_{2 \varepsilon}=0
\end{aligned}
$$

where $\Gamma_{2 \varepsilon}$ is the boundary of the auxiliary domain $\Omega_{2 \varepsilon}$. Passing to the limit as $\varepsilon \rightarrow 0$ in identity (3.34), in view of homogeneous initial boundary conditions $(3.31)-(3.32)$, we arrive at the identity

$$
\begin{aligned}
\int_{0}^{h} v_{x}^{2}(r, y) d y & +\int_{0}^{r} v^{2}(x, h) d x+2 \int_{\Omega_{2}}\left[3 \mu_{1}+a_{2}(x, y)\right] v_{x}^{2} d \Omega_{2} \\
- & \int_{\Omega_{2}}\left[2 \mu_{1}^{3}+2 \mu_{1}^{2} a_{2}(x, y)-2 \mu_{1}\left(a_{2 x}(x, y)-a_{1}(x, y)\right)\right. \\
& \left.+a_{2 x x}(x, y)-a_{1 x}(x, y)+2 a_{0}(x, y)-2 \mu_{2}\right] v^{2} d \Omega_{2}=0 .
\end{aligned}
$$
that

Bearing in mind conditions (3.1), we choose the parameters $\mu_{1}$ and $\mu_{2}$ in identity (3.35) so

$$
\begin{aligned}
\mu_{1}>\frac{1}{3} \max _{(x, y) \in \bar{\Omega}_{2}}\left(\left|a_{2}(x, y)\right|\right), \\
\mu_{2}>\frac{1}{2} \max _{(x, y) \in \bar{\Omega}_{2}}\left[2 \mu_{1}^{3}+2 \mu_{1}^{2}\left|a_{2}(x, y)\right|+2 \mu_{1}\left(\left|a_{2 x}(x, y)\right|+\left|a_{1}(x, y)\right|\right)\right. \\
\left.+\left|a_{2 x x}(x, y)\right|+\left|a_{1 x}(x, y)\right|+2\left|a_{0}(x, y)\right|\right] .
\end{aligned}
$$


It is easy to observe that under such choice of the parameters $\mu_{1}$ and $\mu_{2}$, identity (3.35) can hold if and only if $v(x, y) \equiv 0$ in each point of the closure $\bar{\Omega}_{2}$ that contradicts the assumption that $v(x, y) \neq 0$. The obtained contradiction shows that $u(x, y) \equiv 0$ everywhere in $\bar{\Omega}_{2}$. That is, under assumptions (3.1)-(3.4), the solutions to Problem 1 for equation (1.1) is unique in the required class. The proof is complete.

\section{Theorem on solvability of Problem 1}

Theorem 4.1. Under assumptions (3.1)-(3.4), Problem 1 is solvable.

Proof. Indeed, by the above obtained fundamental relations $(3.10)$ and $(3.22)$, we arrive at the following system of equations for the functions $\tau(x)$ and $\nu(x)$ :

$$
\left\{\begin{array}{l}
\nu(x)=\gamma_{1} D_{r x}^{1-(\alpha+\beta)} \tau(t)-\gamma_{2}(r-x)^{\beta} D_{r x}^{1-\alpha} \psi\left(\frac{r+t}{2}\right), \\
\nu(x)=\tau^{\prime \prime \prime}(x)+a_{2}(x, 0) \tau^{\prime \prime}(x)+a_{1}(x, 0) \tau^{\prime}(x)+a_{0}(x, 0) \tau(x),
\end{array}\right.
$$

and hence, for the function $\tau(x)$, we arrive at the problem on finding a regular solution for the equation

$$
\begin{aligned}
\tau^{\prime \prime \prime}(x) & +a_{2}(x, 0) \tau^{\prime \prime}(x)+a_{1}(x, 0) \tau^{\prime}(x)-\gamma_{1} D_{r x}^{1-\alpha-\beta} \tau(t)+a_{0}(x, 0) \tau(x) \\
& =-\gamma_{2}(r-x)^{\beta} D_{r x}^{1-\alpha} \psi\left(\frac{r+t}{2}\right)
\end{aligned}
$$

satisfying conditions (3.23).

Solving problem 3.23 for equation $(4.2)$ is equivalent to solving the integral equation

$$
\begin{aligned}
\tau(x)=-\frac{1}{2 r^{2}}\{2 & \int_{0}^{r} K(x, t) \tau(t) d t-2(r-x)\left[r+x+r x a_{2}(0,0)\right] \varphi_{1}(0) \\
& -2 r x(r-x) \varphi_{2}(0)-2 x^{2} \varphi_{3}(0)+x^{2} \int_{x}^{r}(r-t)^{2} f(t) d t \\
& \left.-(r-x) \int_{0}^{x} t(r t+x t-2 r x) f(t) d t\right\}
\end{aligned}
$$

where

$$
\begin{aligned}
K(x, t)= & \begin{cases}(r-x)\left[(r+x) L(0, t)+r x L_{x}(0, t)\right]-r^{2} L(x, t), & 0 \leqslant x<t, \\
(r-x)\left[(r+x) L(0, t)+r x L_{x}(0, t)\right], & t<x \leqslant r,\end{cases} \\
L(x, t)= & a_{2}(t, 0)+(t-x)\left[2 a_{2}^{\prime}(t, 0)-a_{1}(t, 0)\right]+\frac{(t-x)^{2}}{2}\left[a_{2}^{\prime \prime}(t, 0)-a_{1}^{\prime}(t, 0)+a_{0}(t, 0)\right] \\
& -\frac{\gamma_{1}}{\Gamma(\alpha+\beta+2)}(t-x)^{\alpha+\beta+2} .
\end{aligned}
$$

Due to properties (3.1) of given coefficients $a_{i}(x, y), i=\overline{0,2}$, in equation $(1.1)$, as well as due to the properties of the given functions $\varphi_{1}(y), \varphi_{2}(y), \varphi_{3}(y), \psi(x)$, we conclude that equation (4.3) is the second kind integral Fredholm equation with a kernel $K(x, t) \in L_{2}([0, r] \times[0, r])$ and the right hand side in $C^{1}[0, r]$. The unique solvability of equation $(4.3)$ is implied by the uniqueness of solution to Problem 1. In accordance with the general theory of Fredholm integral equation, the solution $\tau=\tau(x)$ to equation (4.3) is written out in terms of the resolvent $R(x, t)$ of the kernel $K(x, t)$ and the resolvent $R(x, t)$, as well as the kernel $K(x, t)$, belong to the class $L_{2}([0, r] \times[0, r])$. The solution $\tau=\tau(x)$ to equation $(4.3)$ belongs to the class $\left.C[0, r] \cup C^{3}\right] 0, r[$ 
since the right hand side of equation 4.3 belongs to $C^{1}[0, r]$. By the found function $\tau(x)$ we can find also the function $\nu(x)$ via fundamental relations (3.12), (3.14), (3.16), (3.22).

As the coefficients $a_{i}(x, 0), i=\overline{0,2}$, of equation 4.2 are real constant, the solution to problem (3.23), (4.2), and hence, to integral equation (4.3), is written out explicitly.

Indeed, let us find the solution to problem 3.23 for equation 4.2 in the case when $a_{i}(x, 0)=$ $a_{i}=$ const, $i=\overline{0,2}$. In order to do it, we replace the variable $x$ by $r-x$ in equation (4.2). At that, for the function $\tau(r-x)$ we obtain the problem:

$$
\begin{gathered}
-\tau^{\prime \prime \prime}(r-x)+a_{2} \tau^{\prime \prime}(r-x)-a_{1} \tau^{\prime}(r-x)-\gamma_{1} D_{0 x}^{1-\alpha-\beta} \tau(r-t)+a_{0} \tau(r-x) \\
=-\gamma_{2} x^{\beta} D_{0 x}^{1-\alpha} \psi\left(\frac{2 r-t}{2}\right), \\
\left.\tau(r-x)\right|_{x=r}=\varphi_{1}(0),\left.\quad \tau^{\prime}(r-x)\right|_{x=r}=-\varphi_{2}(0),\left.\quad \tau(r-x)\right|_{x=0}=\varphi_{3}(0) .
\end{gathered}
$$

Denoting $\tau(r-x)=g(x)$, by 4.4 we arrive at the equation for $g(x)$ :

$$
g^{\prime \prime \prime}(x)-a_{2} g^{\prime \prime}(x)+a_{1} g^{\prime}(x)+\gamma_{1} D_{0 x}^{1-\alpha-\beta} g(t)-a_{0} g(x)=f(x),
$$

where $f(x)=\gamma_{2} x^{\beta} D_{0 x}^{1-\alpha} \psi\left(\frac{2 r-t}{2}\right)$.

Applying the operator $D_{0 x}^{-3}$ to the both sides of equation 4.6 , we arrive at an integral equation equivalent to equation (4.6)

$$
\begin{aligned}
g(x) & -\int_{0}^{x}\left[a_{2}-a_{1}(x-t)+\frac{a_{0}}{2}(x-t)^{2}-\frac{\gamma_{1}}{\Gamma(\alpha+\beta+2)}(x-t)^{\alpha+\beta+1}\right] g(t) d t \\
& =c_{1} x^{2}+c_{2} x+c_{3}+\frac{1}{2} \int_{0}^{x}(x-t)^{2} f(t) d t,
\end{aligned}
$$

where $c_{1}, c_{2}, c_{3}$ are unknown constants.

Equation 4.7 belongs to the class of second kind Volterra equation of convolution type. Employing the definition of the convolution of two functions, we rewrite equation (4.7) as

$$
\begin{aligned}
g(x) & -a_{2}(1 * g(x))+a_{1}(x * g(x))-\frac{a_{0}}{2}\left(x^{2} * g(x)\right)+\frac{\gamma_{1}}{\Gamma(\alpha+\beta+2)}\left(x^{\alpha+\beta+1} * g(x)\right) \\
& =\frac{\gamma_{2}}{2}\left(x^{2} * f(x)\right)+c_{1} x^{2}+c_{2} x+c_{3},
\end{aligned}
$$

where

$$
g_{1}(x) * g_{2}(x)=\int_{0}^{x} g_{1}(x-t) g_{2}(t) d t=\int_{0}^{x} g_{1}(t) g_{2}(x-t) d t
$$

is the convolution of functions $g_{1}(x)$ and $g_{2}(x)$.

Let $G(p)$ and $F(p)$ in equation $(4.8)$ be the images of the functions $g(x)$ and $f(x)$, respectively, that is,

$$
g(x) \risingdotseq G(p), \quad f(x) \risingdotseq F(p) .
$$

Then, applying the Laplace transform to equation (4.8), using the linearity and the multiplication theorem, we arrive at the following equation for $G(p)$

$$
G(p)\left[1-\frac{a_{2}}{p}+\frac{a_{1}}{p^{2}}-\frac{a_{0}}{p^{3}}+\frac{\gamma_{1}}{p^{\alpha+\beta+2}}\right]=\frac{F(p)}{p^{3}}+\frac{2 c_{1}}{p^{3}}+\frac{c_{2}}{p^{2}}+\frac{c_{3}}{p},
$$

which implies

$$
G(p)=\frac{F(p)+2 c_{1}+c_{2} p+c_{3} p^{2}}{p^{3} \Delta(p)}
$$

where $\Delta(p)=1-a_{2} p^{-1}+a_{1} p^{-2}+\gamma_{1} p^{-\alpha-\beta-2}-a_{0} p^{-3}$. 
For sufficiently large values of the parameter $p$ the identity

$$
\int_{0}^{\infty} e^{-\Delta(p) s} d s=\frac{1}{\Delta(p)}
$$

holds true. Hence, we can rewrite 4.9 as

$$
G(p)=\int_{0}^{\infty} e^{-\Delta(p) s}\left[F(p) p^{-3}+2 c_{1} p^{-3}+c_{2} p^{-2}+c_{3} p^{-1}\right] d s .
$$

Let us find the inverse Laplace transform. First of all we observe that

$$
p^{-\mu} e^{z p^{-\beta}} \fallingdotseq x^{\mu-1} \phi\left(\beta, \mu ; z x^{\beta}\right),
$$

where $\phi(\xi, \eta ; z)=\sum_{n=0}^{\infty} \frac{z^{n}}{n ! \Gamma(n \xi+\eta)}$ is the Wright function [34].

Employing the formula $g_{1}(p) g_{2}(p) \fallingdotseq q_{1}(x) * q_{2}(x)$, by 4.10 we find

$$
\begin{aligned}
g(x)= & \int_{0}^{\infty} e^{-s}\left\{f(x) *\left[x^{-1 / 4} \phi\left(1,3 / 4 ; a_{2} x s\right)\right] *\left[x^{-1 / 4} \phi\left(2,3 / 4 ;-a_{1} x^{2} s\right)\right]\right. \\
& \left.*\left[x^{-1 / 4} \phi\left(\alpha+\beta+2,3 / 4 ;-\gamma_{1} x^{\alpha+\beta+2} s\right)\right] *\left[x^{-1 / 4} \phi\left(3,3 / 4 ; a_{0} x^{3} s\right)\right]\right\} d s \\
& +2 c_{1} \int_{0}^{\infty} e^{-s}\left\{\left[x^{-1 / 4} \phi\left(1,3 / 4 ; a_{2} x s\right)\right] *\left[x^{-1 / 4} \phi\left(2,3 / 4 ;-a_{1} x^{2} s\right)\right]\right. \\
& \left.*\left[x^{-1 / 4} \phi\left(\alpha+\beta+2,3 / 4 ;-\gamma_{1} x^{\alpha+\beta+2} s\right)\right] *\left[x^{-1 / 4} \phi\left(3,3 / 4 ; a_{0} x^{3} s\right)\right]\right\} d s \\
+ & c_{2}^{\infty} e^{-s}\left\{\left[x^{-1 / 2} \phi\left(1,1 / 2 ; a_{2} x s\right)\right] *\left[x^{-1 / 2} \phi\left(2,1 / 2 ;-a_{1} x^{2} s\right)\right]\right. \\
& \left.*\left[x^{-1 / 2} \phi\left(\alpha+\beta+2,1 / 2 ;-\gamma_{1} x^{\alpha+\beta+2} s\right)\right] *\left[x^{-1 / 2} \phi\left(3,1 / 2 ; a_{0} x^{3} s\right)\right]\right\} d s \\
& \int_{0}^{\infty} e^{-s}\left\{\left[x^{-3 / 4} \phi\left(1,1 / 4 ; a_{2} x s\right)\right] *\left[x^{-3 / 4} \phi\left(2,1 / 4 ;-a_{1} x^{2} s\right)\right]\right. \\
& \left.*\left[x^{-3 / 4} \phi\left(\alpha+\beta+2,1 / 4 ;-\gamma_{1} x^{\alpha+\beta+2} s\right)\right] *\left[x^{-3 / 4} \phi\left(3,1 / 4 ; a_{0} x^{3} s\right)\right]\right\} d s .
\end{aligned}
$$

We employ the following notations [35]:

$$
\begin{aligned}
& S_{m}^{\mu}\left(x ; z_{1}, \ldots, z_{m} ; \beta_{1}, \ldots, \beta_{m}\right)=h_{1}(x) * h_{2}(x) * \ldots * h_{m}(x) \\
& G_{m}^{\mu}(x ; \lambda ; \beta) \equiv G_{m}^{\mu}\left(x ; \lambda_{1}, \ldots, \lambda_{m} ; \beta_{1}, \ldots, \beta_{m}\right)=\int_{0}^{\infty} e^{-s} S_{m}^{\mu}\left(x ; \lambda_{1} s, \ldots, \lambda_{m} s ; \beta_{1}, \ldots, \beta_{m}\right) d s,
\end{aligned}
$$

where $h_{k}(x)=x^{\mu_{k}-1} \phi\left(\beta_{k}, \mu_{k} ; z_{k} x^{\beta_{k}}\right), k=\overline{1, m} ; \mu=\sum_{k=1}^{m} \mu_{k}$. In terms of the above notations, representation 4.11 can be rewritten as

$$
\begin{aligned}
g(x)= & \int_{0}^{\infty} e^{-s}\left[f(x) * S_{4}^{3}\left(x ; a_{2} s,-a_{1} s,-\gamma_{1} s, a_{0} s ; 1,2, \alpha+\beta+2,3\right)\right] d s \\
& +2 c_{1} \int_{0}^{\infty} e^{-s} S_{4}^{3}\left(x ; a_{2} s,-a_{1} s,-\gamma_{1} s, a_{0} s ; 1,2, \alpha+\beta+2,3\right) d s
\end{aligned}
$$




$$
\begin{aligned}
& +c_{2} \int_{0}^{\infty} e^{-s} S_{4}^{2}\left(x ; a_{2} s,-a_{1} s,-\gamma_{1} s, a_{0} s ; 1,2, \alpha+\beta+2,3\right) d s \\
& +c_{3} \int_{0}^{\infty} e^{-s} S_{4}^{1}\left(x ; a_{2} s,-a_{1} s,-\gamma_{1} s, a_{0} s ; 1,2, \alpha+\beta+2,3\right) d s
\end{aligned}
$$

which implies

$$
\begin{aligned}
g(x)= & 2 c_{1} G_{4}^{3}\left(x ; a_{2},-a_{1},-\gamma_{1}, a_{0} ; 1,2, \alpha+\beta+2,3\right) \\
& +c_{2} G_{4}^{2}\left(x ; a_{2},-a_{1},-\gamma_{1}, a_{0} ; 1,2, \alpha+\beta+2,3\right) \\
& +c_{3} G_{4}^{1}\left(x ; a_{2},-a_{1},-\gamma_{1}, a_{0} ; 1,2, \alpha+\beta+2,3\right) \\
& +\int_{0}^{x} f(t) G_{4}^{3}\left(x-t ; a_{2},-a_{1},-\gamma_{1}, a_{0} ; 1,2, \alpha+\beta+2,3\right) d t .
\end{aligned}
$$

Thus,

$$
\tau(r-x)=2 c_{1} G_{4}^{3}(x ; \mathbf{a} ; \mathbf{b})+c_{2} G_{4}^{2}(x ; \mathbf{a} ; \mathbf{b})+c_{3} G_{4}^{1}(x ; \mathbf{a} ; \mathbf{b})+\int_{0}^{x} f(t) G_{4}^{3}(x-t ; \mathbf{a} ; \mathbf{b}) d t
$$

where $\mathbf{a}=\left(a_{2},-a_{1},-\gamma_{1}, a_{0}\right), \mathbf{b}=(1,2, \alpha+\beta+2,3)$. Redenoting $r-x$ by $x$ in the last identity and changing the variable $s=r-t$ in the integral, we obtain

$$
\begin{aligned}
\tau(x)= & 2 c_{1} G_{4}^{3}(r-x ; \mathbf{a} ; \mathbf{b})+c_{2} G_{4}^{2}(r-x ; \mathbf{a} ; \mathbf{b})+c_{3} G_{4}^{1}(r-x ; \mathbf{a} ; \mathbf{b}) \\
& +\int_{x}^{r} f(r-s) G_{4}^{3}(s-x ; \mathbf{a} ; \mathbf{b}) d s .
\end{aligned}
$$

The function $G_{m}^{\mu}(x)$ possesses the properties [35]:

$$
\begin{aligned}
& D_{0 x}^{\nu} G_{m}^{\mu}(t ; \mathbf{a} ; \mathbf{b})=G_{m}^{\mu-\nu}(t ; \mathbf{a} ; \mathbf{b}), \quad \mu>\nu ; \\
& G_{m}^{\mu}(x ; \mathbf{a} ; \mathbf{b})=\frac{x^{\mu-1}}{\Gamma(\mu)}+\sum_{i=1}^{m j} \lambda_{i} D_{0 x}^{-\beta_{i}} G_{m}^{\mu}(t ; \mathbf{a} ; \mathbf{b}) .
\end{aligned}
$$
find

Employing properties 4.13), 4.14) of the function $G_{m}^{\mu}(x ; \mathbf{a} ; \mathbf{b})$, by representation 4.12 we

$$
\begin{aligned}
\tau^{\prime}(x)= & -c_{3}\left[a_{2} G_{4}^{1}(r-x ; \mathbf{a} ; \mathbf{b})-a_{1} G_{4}^{2}(r-x ; \mathbf{a} ; \mathbf{b})-\gamma_{1} G_{4}^{\alpha+\beta+2}(r-x ; \mathbf{a} ; \mathbf{b})\right. \\
& \left.+a_{0} G_{4}^{3}(r-x ; \mathbf{a} ; \mathbf{b})\right]-2 c_{1} G_{4}^{2}(r-x ; \mathbf{a} ; \mathbf{b})-c_{2} G_{4}^{1}(r-x ; \mathbf{a} ; \mathbf{b}) \\
& -\int_{x}^{r} f(r-s) G_{4}^{2}(s-x ; \mathbf{a} ; \mathbf{b}) d s .
\end{aligned}
$$

Let us find the unknown constants $c_{1}, c_{2}, c_{3}$ involved in (4.12). Substituting (4.12) into the latter condition in (3.23), we immediately find

$$
\tau(r)=c_{3}=\varphi_{3}(0)
$$


Substituting then $(4.12)$ into first two conditions in 3.23$)$, we arrive at the following system of linear algebraic equations:

$$
\left\{\begin{aligned}
2 c_{1} G_{4}^{3}(r ; \mathbf{a} ; \mathbf{b})+c_{2} G_{4}^{2}(r ; \mathbf{a} ; \mathbf{b})= & \varphi_{1}(0)-\varphi_{3}(0) G_{4}^{1}(r ; \mathbf{a} ; \mathbf{b}) \\
& -\int_{0}^{r} f(r-s) G_{4}^{3}(s ; \mathbf{a} ; \mathbf{b}) d s \\
2 c_{1} G_{4}^{2}(r ; \mathbf{a} ; \mathbf{b})+c_{2} G_{4}^{1}(r ; \mathbf{a} ; \mathbf{b})= & \varphi_{2}(0)-\varphi_{3}(0)\left[a_{2} G_{4}^{1}(r ; \mathbf{a} ; \mathbf{b})-a_{1} G_{4}^{2}(r ; \mathbf{a} ; \mathbf{b})\right. \\
& \left.-\gamma_{1} G_{4}^{\alpha+\beta+2}(r ; \mathbf{a} ; \mathbf{b})+a_{0} G_{4}^{3}(r ; \mathbf{a} ; \mathbf{b})\right] \\
& -\int_{0}^{r} f(r-s) G_{4}^{2}(s ; \mathbf{a} ; \mathbf{b}) d s .
\end{aligned}\right.
$$

Solving system (4.16), we find

$$
c_{1}=\frac{\Delta_{1}}{\Delta}, \quad c_{2}=\frac{\Delta_{2}}{\Delta}
$$

where

$$
\begin{aligned}
\Delta= & 2\left[G_{4}^{1}(r ; \mathbf{a} ; \mathbf{b}) G_{4}^{3}(r ; \mathbf{a} ; \mathbf{b})-\left(G_{4}^{2}(r ; \mathbf{a} ; \mathbf{b})\right)^{2}\right], \\
\Delta_{1}= & \int_{0}^{r} f(r-s)\left[G_{4}^{2}(r ; \mathbf{a} ; \mathbf{b}) G_{4}^{2}(s ; \mathbf{a} ; \mathbf{b})-G_{4}^{1}(r ; \mathbf{a} ; \mathbf{b}) G_{4}^{3}(s ; \mathbf{a} ; \mathbf{b})\right] d s \\
& +G_{4}^{1}(r ; \mathbf{a} ; \mathbf{b})\left[\varphi_{1}(0)-\varphi_{3}(0) G_{4}^{1}(r ; \mathbf{a} ; \mathbf{b})\right] \\
& -G_{4}^{2}(r ; \mathbf{a} ; \mathbf{b}) \cdot\left[\varphi_{2}(0)-\varphi_{3}(0)\left(a_{2} G_{4}^{1}(r ; \mathbf{a} ; \mathbf{b})-a_{1} G_{4}^{2}(r ; \mathbf{a} ; \mathbf{b})\right.\right. \\
& \left.\left.-\gamma_{1} G_{4}^{\alpha+\beta+2}(r ; \mathbf{a} ; \mathbf{b})+a_{0} G_{4}^{3}(r ; \mathbf{a} ; \mathbf{b})\right)\right], \\
& r \\
\Delta_{2} & \int_{0} f(r-s)\left[G_{4}^{3}(s ; \mathbf{a} ; \mathbf{b}) G_{4}^{3}(s ; \mathbf{a} ; \mathbf{b})-G_{4}^{3}(s ; \mathbf{a} ; \mathbf{b}) G_{4}^{3}(s ; \mathbf{a} ; \mathbf{b})\right] d s \\
& -2 G_{4}^{2}(r ; \mathbf{a} ; \mathbf{b})\left[\varphi_{1}(0)-\varphi_{3}(0) G_{4}^{1}(r ; \mathbf{a} ; \mathbf{b})\right]+2 G_{4}^{3}(r ; \mathbf{a} ; \mathbf{b}) \\
& \cdot\left[\varphi_{2}(0)-\varphi_{3}(0)\left(a_{2} G_{4}^{1}(r ; \mathbf{a} ; \mathbf{b})-a_{1} G_{4}^{2}(r ; \mathbf{a} ; \mathbf{b})-\gamma_{1} G_{4}^{\alpha+\beta+2}(r ; \mathbf{a} ; \mathbf{b})+a_{0} G_{4}^{3}(r ; \mathbf{a} ; \mathbf{b})\right)\right] .
\end{aligned}
$$

It follows from the above proven theorem on the uniqueness of solution to Problem 1 that the determinant

$$
\Delta=2\left[G_{4}^{1}(r ; \mathbf{a} ; \mathbf{b}) G_{4}^{3}(r ; \mathbf{a} ; \mathbf{b})-\left(G_{4}^{2}(r ; \mathbf{a} ; \mathbf{b})\right)^{2}\right]
$$

of system 4.16) is non-zero and therefore, formula 4.12, where the constants $c_{1}, c_{2}$ are calculated by formulae (4.17), $c_{3}=\varphi_{3}(0)$, gives the representation for the unique solution to problem (3.23) for equation (4.2) as the coefficients $a_{0}, a_{1}, a_{2}$ of equation (4.2) are constant and $-m / 2 \leqslant a<m / 2$.

If $a=m / 2$, as the coefficients $a_{0}, a_{1}, a_{2}$ are constant, by relations $(3.16)$ and $(3.22)$ we arrive at the following problem for the sought function $\tau=\tau(x)$

$$
\begin{aligned}
& \tau^{\prime \prime \prime}(x)+a_{2} \tau^{\prime \prime}(x)+a_{1} \tau^{\prime}(x)+a_{0} \tau(x)=(2-2 \beta)^{-\beta}(r-x)^{\beta} \psi^{\prime}\left(\frac{r+x}{2}\right), \\
& \tau(0)=\varphi_{1}(0), \quad \tau^{\prime}(0)=\varphi_{2}(0), \quad \tau(r)=\varphi_{3}(0) .
\end{aligned}
$$


The solution to problem 4.18), 4.19) is written out explicitly by the formula

$$
\tau(x)=\int_{0}^{r} G(x, t) F(t) d t+\frac{x^{2}}{r^{2}}\left[\varphi_{3}(0)-r \varphi_{2}(0)-\varphi_{1}(0)\right]+\varphi_{2}(0) x+\varphi_{1}(0),
$$

where

$$
\begin{aligned}
F(x)= & (2-2 \beta)^{-\beta}(r-x)^{\beta} \psi^{\prime}\left(\frac{r+x}{2}\right)-a_{0} \varphi_{1}(0)-\left(a_{0} x+a_{1}\right) \varphi_{2}(0) \\
& -\frac{\varphi_{3}(0)-r \varphi_{2}(0)-\varphi_{1}(0)}{r^{2}}\left(2 a_{2}+2 a_{1} x+a_{0}\right)
\end{aligned}
$$

$G(x, t)$ is the Green function of problem (4.18), 4.19) constructed in work [25].

Once the functions $\tau=\tau(x)$ and $\nu=\nu(x)$ are found, the solutions to Problem 1 in the domain $\Omega_{1}$ is determined as the solution to Cauchy problem $(3.5),(3.6)$ for equation $(1.2)$ and is written out by one of formulae (3.7), (3.13) or $(3.15)$, while in the domain $\Omega_{2}$ we arrive at the problem on finding a regular solution to equation (1.3) satisfying conditions $(2.1)$ and $u(x, 0)=\tau(x)$; this problem was studied in works [14], [15].

\section{BIBLIOGRAPHY}

1. A.V. Bitsadze. Mixed type equations. Izd-vo AN SSSR, Moscow (1949). (in Russian).

2. A.V. Lykov. Application of methods of irreversible processes thermodynamics to studying the heat and mass exchange // Inzhenerno-fizicheskii zhurn. 9:3, 287-304 (1955). (in Russian).

3. A.M. Nakhushev. Equations of mathematical biology. Vysshaya shkola, Moscow (1995). (in Russian).

4. A.M. Nakhushev. Fractional calculus and its applications. Fizmatlit, Moscow (2003). (in Russian).

5. L. Bers. Mathematical aspects of subsonic and transonic gas dynamics. John Wiley \& Sons, New York (1958).

6. F.I. Frankl. Selected works on gas dynamics. Nauka, Moscow (1973). (in Russian).

7. T.S. Kal'menov. A criterion for the continuity of a solution of Goursat's problem for a degenerate equation // Differ. Uravn. 7:1, 178-181 (1971). (in Russian).

8. T.S. Kal'menov. Darboux's problem for a degenerate equation // Differ. Uravn. 10:1, 59-68 (1974). [Diff. Equat. 10:1, 41-47 (1975).]

9. T.S. Kal'menov. A criterion for the continuity of a solution of Goursat's problem for a degenerate equation // Differ. Uravn. 8:1, 41-55 (1972). (in Russian).

10. M.M. Smirnov. Degenerate hyperbolic equations. Vyshejshaya shkola, Minsk (1977). (in Russian).

11. O.A. Repin. Boundary value problems with a shift for hyperbolic and mixed type equations. Izd-vo Saratov. Univ., Saratov (1992). (in Russian).

12. A.M. Nakhushev. Problems with shifts for partial differential equations. Nauka, Moscow (2006). (in Russian).

13. T.Sh. Kal'menov. To the theory of initial boundary value problems for differential equations. Series of scientific works by T.Sh. Kal'menov. Inst. Math. Math. Model., Almaty (2013). (in Russian).

14. T.D. Dzhuraev. Boundary value problems for equations of mixed and mixed-composite types. FAN, Tashkent (1979). (in Russian).

15. L. Cattabriga. Un Problema al contorno per una equazione parabolica di ordine dispari // Ann. del. Scu. Norm. Super. Pisa. 13:2, 163-203 (1959).

16. Yu. Irgashev. Some boundary value problems for third order equations with multiple characteristics // in Collection of scientific works "Boundary value problems for differential equations and their applications". FAN, Tashkent, 17-31 (1976). (in Russian).

17. T.D. Dzhuraev, S. Abdinazarov. Boundary value problems of Bitsadze-Samarsky problem type for third order equations with multiple characteristics // Izv. AN Uzbek. SSR. 1, 8-11 (1981). (in Russian). 
18. S. Abdinazarov. General boundary value problems for an equation of third order with multiple characteristics. // Differ. Uravn. 17:1, 3-12 (1981). [Differ. Equat. 17:1, 1-8 (1981).]

19. I.M. Gel'fand. Some questions of analysis and differential equations // Uspekhi Matem. Nauk. 14:3(87), 3-19 (1959). (in Russian).

20. A.V. Bitsadze. Mixed type equations. Izd-vo AN SSSR, Moscow (1959). (in Russian).

21. A.V. Bitsadze. To the theory of one class of mixed type equations // in book "Some problems of mathematics and mechanics", Nauka, Leningrad, 112-119 (1970). (in Russian).

22. O.A. Repin, S.K. Kumykova. Problem with shift for the third-order equation with discontinuous coefficients // Vestn. Samar. Gos. Tekhn. Univ. Ser. Fiz.-Mat. Nauki. 4(29), 17-25 (2012). (in Russian).

23. O.A. Repin, S.K. Kumykova. Nonlocal problem for a equation of mixed type of third order with generalized operators of fractional integro-differentiation of arbitrary order // Vestn. Samar. Gos. Tekhn. Univ. Ser. Fiz.-Mat. Nauki. 4(25), 25-36 (2011). (in Russian).

24. V.A. Eleev, S.K. Kumykova. Interior boundary value problem for mixed type third order equation with multiple characteristics // Izv. Kabardino-Balkar Scientific Center of RAS. 5, 5-14 (2010). (in Russian).

25. Zh.A. Balkizov. Local and nonlocal value boundary problems for a third-order mixed-type equation equipped with Tricomi operator in its hyperbolic part // Vestn. Samar. Gos. Tekhn. Univ. Ser. Fiz.-Mat. Nauki. 2(17), 21-28 (2008). (in Russian).

26. Zh.A. Balkizov. Boundary value problems for a mixed type third order equations with Gellerstedt operator in the hyperbolic part // Izv. Kabardino-Balkar. Gos. Univ. 1:1, 21-33 (2011). (in Russian).

27. Zh.A. Balkizov. Analouge of Tricomi problem for a third order parabolic-hyperbolic equation with Gellerstedt operator in the hyperbolicity domain // Dokl. Adygskoi (Cherkesskoi) Mezhdunar. Akad. Nauk. 16:2, 20-27 (2014). (in Russian).

28. Zh.A. Balkizov. Nonlocal boundary value problem for a model third order parabolic-hyperbolic equation // Dokl. Adygskoi (Cherkesskoi) Mezhdunar. Akad. Nauk. 17:4, 9-20 (2015). (in Russian).

29. L.A. Zolina. On a boundary value problem for a model equation of hyperbolo-parabolic type // Zhurn. Vychisl. Matem. Matem. Fiz. 6:6, 991-1001 (1966). (in Russian).

30. H.G. Bzkikhatlov, A.M. Nakhushev. A boundary value problem for a mixed equation of parabolichyperbolic type // Dokl. Akad. Nauk SSSR. 183:2, 261-264 (1968). [Sov. Math., Dokl. 9, 1349-1352 (1968).]

31. S.G. Samko, A.A. Kilbas, O.I. Marichev. Fractional integrals and derivatives: theory and applications. Nauka i Tekhnika, Minsk (1987). [Gordon and Breach, New York (1993).]

32. A.A. Alikhanov. A priori estimates for solutions of boundary value problems for fractional-order equations // Differ. Uravn. 46:5, 658-664 (2010). [Diff. Equat. 46:5, 660-666 (2010).]

33. A.M. Nakhushev. A contribution to the theory of linear boundary-value problems for second-order mixed hyperbolic-parabolic equations // Differ. Uravn. 14:1, 56-73 (1978). [Diff. Equat. 14:1, 46-51 (1978).]

34. E.M. Wright. The generalized Bessel function of order greater than one // Quart. J. Math. Oxford Ser. 11:1, 36-48 (1940).

35. A.V. Pskhu. Initial-value problem for a linear ordinary differential equation of noninteger order // Matem. Sborn. 202:4, 111-122 (2011). [Sb. Math. 202:4, 571-582 (2011).]

Zhiraslan Anatol'evich Balkizov,

Institute of Applied Mathematics and Automation,

Kabardino-Balkar Scientific Center, RAS,

Shortanova str. 89-a,

360005, Nalchik, Russia

E-mail: Giraslan@yandex.ru 